\title{
El consumo abusivo de alcohol en inmigrantes latinoamericanos participantes en programas de intervención para maltratadores: importancia de la adherencia al tratamiento
}

\section{Alcohol Abuse in Latin American Immigrants Participating in Intervention Programs for Abusers: the Importance of Adherence to Treatment}

Recepción: 24 Abril 2016 | Aprobación: 03 Julio 2016

\author{
Marisol Lila** \\ Universidad de Valencia, España \\ Enrique Gracia \\ Universidad de Valencia, España \\ Alba Catalá-Miñana \\ Universidad de Valencia, España \\ Faraj A. Santirso \\ Universidad de Valenci, España \\ Ángel Romero-Martínez \\ Universidad de Valencia, España
}

\footnotetext{
**Correo electrónico: marisol.lila@uv.es; enrique.gracia@uv.es; alba.catala@uv.es; faraj.santirso@uv.es; angel.romero@uv.es
}

\section{RESUMEN}

En este estudio, se analizan las tasas de abandono del tratamiento en hombres inmigrantes latinoamericanos con problemas de consumo de alcohol (PCA) derivados a programas de intervención con maltratadores, y se explora si aquellos que consiguen finalizar la intervención se diferencian de los que no tienen PCA en variables resultado de la intervención. La muestra la componen 107 hombres latinoamericanos condenados por violencia contra la mujer. Al contrario de lo esperado, no se confirman mayores tasas de abandono entre los participantes con PCA. Independientemente de tener o no PCA, los participantes que finalizaron el programa mostraron mejoría en todas las variables objetivo de la intervención analizadas, incluida la reducción del consumo de alcohol entre aquellos participantes con PCA.

Palabras clave

alcohol, abandono del tratamiento, latinoamericanos, programas de intervención con maltratadores, violencia contra la mujer.

\begin{abstract}
This study analyzes dropout rates among Latin-American immigrants with alcohol abuse problems (AAP) participating in a batterer intervention program, and whether those perpetrators who finalize the intervention benefit the same as those participants without AAP in a number of program outcomes. The sample was 107 Latin-American immigrants convicted for intimate partner violence perpetration. Contrary
\end{abstract}


Marisol Lila, Enrique Gracia, Alba Catalá-Miñana, Faraj A. Santirso, Et al.

to what was expected, there were not higher dropout rates among participants with AAP. Participants who finalized the intervention program, however, showed improvements in all outcomes variables explored regardless of whether they had AAP, including a reduction of alcohol abuse among participants with AAP.

Keywords

alcohol, batterer intervention programs, dropout, Latin Americans, intimate partner violence.

Para citar este artículo: Lila, M., Gracia, E., Catalá-Miñana, A., Santirso, F. A., \& RomeroMartínez, A. (2016). El consumo abusivo de alcohol en inmigrantes latinoamericanos partipantes en programas de intervención para maltratadores: importancia de la adherencia al tratamiento. Universitas Psychologica, 15 (4). http://dx.doi.org/10.11144/Javeriana.upsy15 $-4 . c a a i$

La violencia contra la mujer en las relaciones de pareja (VMRP) ha sido conceptualizada como un problema de salud pública de proporciones epidémicas (Organización Mundial de la Salud, 2013). La VMRP es la forma más común de violencia sufrida por las mujeres; el porcentaje de mujeres que han sufrido violencia física y/ o sexual por parte de su pareja alguna vez en su vida se sitúa en torno al $30 \%$ (Devries et al., 2013; European Union Agency for Fundamental Rights, 2014). Entre las iniciativas que se han adoptado para prevenir este problema, se encuentran los programas de intervención con maltratadores (Babcock, Green, \& Robie, 2004; Gondolf, 2012; Lila, 2013). Desde sus orígenes, se ha enfatizado la necesidad de que estos programas sean evaluados y prueben su eficacia. Sin embargo, la mayoría de estudios de evaluación y los metaanálisis realizados hasta la fecha indican tamaños pequeños del efecto (Arias, Arce, \& Vilariño, 2013; Babcock et al., 2004; Eckhardt et al., 2013; Feder \& Wilson, 2005). Una de las causas de la limitada eficacia de los programas de intervención con maltratadores, señalada reiteradamente en la literatura científica, son las altas tasas de abandono (Crane, Eckhardt, \& Schlauch, 2015). Estas tasas varían, según los estudios, entre el 15 y el $60 \%$ (Bennett, Stoops, Call, \& Flett, 2007;
Rondeau, Brodeur, Brochu, \& Lemire, 2001). Estos datos son especialmente preocupantes si tenemos en cuenta que existe una estrecha relación entre abandono y reincidencia (Jewell \& Wormith, 2010).

Entre los factores predictores del abandono de los programas de intervención con maltratadores, así como de la reincidencia, se encuentra el consumo abusivo de alcohol (Catalá-Miñana, Lila, \& Oliver, 2013; Jewell \& Wormith, 2010; Olver, Stockdale, \& Wormith, 2011). De hecho, el consumo abusivo de alcohol se ha considerado como uno de los principales factores vinculados a la VMRP (Langenderfer, 2013; OMS, 2010). Algunos metaanálisis muestran la relación entre problemas de consumo de alcohol (PCA) y VMRP de forma consistente, indicando que el consumo elevado de alcohol incrementa 4.6 veces la probabilidad de VMRP en comparación con el bajo consumo o la abstinencia (Foran \& O'leary, 2008; Gil-González, Vives-Cases, Álvarez-Dardet, \& Latour-Pérez, 2006). La estrecha relación entre violencia en las relaciones de pareja y PCA se hace evidente, por ejemplo, en el hecho de que cerca del $50 \%$ de los hombres condenados por VMRP derivados a programas de intervención como maltratadores presentan algún tipo de problema vinculado al consumo de alcohol (Arteaga, López-Goñi, \& Fernández-Montalvo, 2015; Crane et al., 2015; Stuart, O'Farrell, \& Temple, 2009). Esta estrecha relación entre VMRP y PCA llevó a la Organización Mundial de la Salud (2010) a recomendar las campañas de prevención del abuso del alcohol como una estrategia para reducir la VMRP.

\section{VPM y PCA en un grupo de riesgo: los inmigrantes latinoamericanos en España}

Aunque la VMRP pueden sufrirla mujeres de cualquier país y condición social, existen algunos colectivos de mujeres especialmente vulnerables (Abramsky et al., 2011; Contreras, 2014; Jewkes, 2002; Sabina, Cuevas, \& Lannen, 2014). En este sentido, algunos estudios sugieren que la 
prevalencia de la VMRP difiere entre distintos grupos étnicos y colectivos de inmigrantes. Por ejemplo, en numerosos estudios se han encontrado tasas más elevadas de VMRP entre inmigrantes latinoamericanos frente a las presentadas por la población autóctona (Cho, Velez-Ortiz, \& Parra-Cardona, 2014; ParraCardona et al., 2013; Vargas, Lila, \& CataláMiñana, 2015; Vaughn, Salas-Wright, CooperSadlo, Maynard, \& Larson, 2014). En España, donde el colectivo de inmigrantes más numeroso es el latinoamericano, un tercio de los casos denunciados por VMRP corresponden a mujeres inmigrantes, de las que casi el $50 \%$ son latinoamericanas (Ministerio de Sanidad, 2011; Observatorio contra la Violencia Doméstica y de Género, 2014). En torno al $25 \%$ de los penados por un delito de VMRP son inmigrantes, siendo los latinoamericanos el colectivo extranjero más numeroso (Carbajosa, Lila, Negredo, \& Pérez, 2011; Echauri, Fernández-Montalvo, Martínez, \& Azkarate, 2013). En este mismo sentido, en una reciente investigación basada en la geolocalización de casos de VMRP en la ciudad de Valencia (España), se señala un mayor riesgo de VMRP, en barrios con una mayor concentración de inmigrantes (Gracia, LópezQuílez, Marco, Lladosa, \& Lila, 2014, 2015).

En relación con el consumo de alcohol, en numerosos estudios, se señala que el colectivo de inmigrantes latinoamericanos presenta niveles más elevados de consumo que otros grupos (Conde \& Herranz, 2004; Tortajada et al., 2008; Unger, 2015). Igualmente, se ha encontrado que muestra actitudes más tolerantes hacia el consumo de alcohol y una menor percepción del riesgo que conlleva su consumo abusivo (Conde \& Herranz, 2004; Sussman, 2005; Tortajada et al., 2008). En este sentido, algunos autores señalan que el consumo de alcohol juega un papel importante en el proceso de socialización de este colectivo (Camacho \& Comas, 2003; Conde \& Herranz, 2004).

El hecho de que el colectivo de inmigrantes latinoamericano condenados por VMRP presente al mismo tiempo mayores tasas de violencia como de consumo de alcohol sugiere necesidades especiales que deberían ser tenidas en cuenta por los profesionales en los programas de intervención con maltratadores. Sin embargo, aunque con alguna excepción (p. ej., Vargas et al., 2015), en España este colectivo apenas ha sido estudiado y poco se conoce de su funcionamiento en los programas de intervención con maltratadores.

\section{El presente estudio}

A pesar de que el PCA es un importante factor de riesgo vinculado a la VMRP y de que existen colectivos que tienen una mayor presencia en los programas de intervención, como es el caso de los inmigrantes latinoamericanos, en la práctica, la mayoría de los programas de intervención con maltratadores no incluyen el tratamiento específico para los PCA dentro de su programación ni están adaptados culturalmente (Carbajosa \& Boira, 2013; Langenderfer, 2013). ¿Qué sucede con aquellos participantes latinoamericanos con PCA que son derivados a estos programas?, ¿Es más probable que abandonen la intervención que aquellos que no tienen PCA? y ¿qué sucede con los que consiguen finalizar la intervención?, ¿obtienen los mismos resultados que aquellos que no tienen PCA? Hasta donde sabemos, no hay todavía ningún estudio que dé respuesta a estas cuestiones.

Este estudio tiene dos objetivos: El primero es determinar si, en un programa de intervención con maltratadores, las tasas de abandono de la intervención son más elevadas en los hombres inmigrantes latinoamericanos con PCA que aquellos sin PCA. Se espera que aquellos participantes con PCA abandonen la intervención con mayor probabilidad, tal y como sucede en investigaciones previas realizadas en el ámbito anglosajón (Daly \& Pelowski, 2000; Olver et al., 2011). El segundo objetivo es analizar si, en comparación con aquellos participantes latinoamericanos que no tienen PCA, los resultados de la intervención son los mismos para los participantes latinoamericanos que, aun teniendo PCA, sí consiguen finalizar la intervención. Como resultados de la intervención 
se tendrán en cuenta la reincidencia informada en los registros oficiales (Bowen, 2011), la valoración del riesgo de reincidencia (AndrésPueyo \& Echeburúa, 2010; Lila, Oliver, CataláMiñana, \& Conchell, 2014; Scott, King, McGinn, \& Hosseini, 2011), la atribución de responsabilidad (i. e., atribución de la responsabilidad de los hechos por los que han sido condenados al sistema legal, a la víctima o a su contexto personal; Gracia \& Tomás, 2014; Henning \& Holdford, 2006; Lila, Gracia, \& Herrero, 2012; Lila, Oliver, Catalá-Miñana, Galiana, \& Gracia, 2014; Scott et al., 2011), las actitudes hacia la VMRP (i. e., gravedad percibida de la VMRP; Gracia, García, \& Lila, 2011; Lila, Gracia, \& García, 2013), las actitudes sexistas (i. e., tanto el sexismo hostil como el sexismo benevolente; Glick, Sakalli-Ugurlu, Ferreira, \& de Souza, 2002; Gracia et al., 2014; Lila, Oliver, Galiana, \& Gracia, 2013) y el ajuste psicosocial (i. e., sintomatología depresiva y apoyo social íntimo; Graham et al., 2011; Lila, Gracia, \& Murgui, 2013; Lila, Oliver, Lorenzo, \& Catalá-Miñana, 2013; Novo, Fariña, Seijó, \& Arce, 2012). Además, se examinará si se ha producido una reducción en el consumo de alcohol al finalizar la intervención (Bennett, 2008).

\section{Método}

\section{Participantes y procedimiento}

La muestra se compone de 107 hombres latinoamericanos residentes en España y penados por VMRP derivados a un programa de intervención con maltratadores. Los datos para este estudio fueron recogidos mediante la administración de una batería de cuestionarios en fase pretratamiento y postratamiento como parte del protocolo del programa de intervención aplicado. El estudio fue aprobado por el Comité de Ética en Investigación Experimental de la Universidad de Valencia y fue llevado a cabo de acuerdo a las normas de la Declaración de Helsinki. Antes de comenzar la recolección de datos, los participantes firmaron un consentimiento informado para la participación en el estudio.

La media de edad de los participantes fue de 34.52 ( $D E=8.31$; rango de 19 a 53 años), todos ellos procedentes de países latinoamericanos (48.6\% de Ecuador, $20.6 \%$ de Bolivia, $14 \%$ de Colombia y el $16.8 \%$ restante de países como Uruguay, Cuba, Argentina, Perú, Santo Domingo, Chile, Brasil, Venezuela, Honduras o Guatemala); el $30.8 \%(n=33)$ estaban casados o en pareja; el $3.7 \%(n=4)$ no contaban con estudios, el $27.1 \%(n=29)$ con estudios primarios; el $54.2 \%(n=58)$ con estudios secundarios y el $15 \%(n=16)$ con estudios universitarios; el $23.4 \%(n=25)$ se encontraban en situación de desempleo y la media de ingresos se situaba entre 6.000 y 12.000 euros/año.

\section{Intervención}

El programa de intervención con hombres penados por VMRP se realizó en medio comunitario en la ciudad de Valencia, España. La duración del programa fue de 9 meses y fue llevado a cabo en 32 sesiones grupales de dos horas de duración, una vez a la semana, dirigidas por dos terapeutas. La fase de evaluación incluía la administración de cuestionarios de autoinforme, test estandarizados, tres entrevistas en profundidad y una valoración del riesgo de reincidencia. La fase de intervención, basada en el modelo ecológico (Heise, 2011), tenía como objetivo incrementar las habilidades y reducir los factores de riesgo a nivel individual (e. g., técnicas de control emocional), nivel interpersonal (e. g., habilidades de comunicación positiva en las relaciones de pareja), nivel situacional (e. g., apoyo social) y nivel sociocultural (e. g., roles de género y actitudes sexistas). La fase de seguimiento tuvo una duración de 18 meses (véase Lila, Oliver, CataláMiñana, \& Conchell, 2014 para una descripción más completa del programa de intervención). 


\section{Instrumentos}

\section{Datos sociodemográficos}

Se recogió información sobre la edad, el estado civil $(1=$ casado o en pareja; $2=$ soltero; 3 = separado $; 4=$ divorciado $; 5=$ viudo), nivel educativo ( $1=\sin$ estudios; $2=$ primarios; 3 = secundarios; $4=$ universitarios), lugar de nacimiento, situación laboral $(1=$ empleado; 2 $=$ desempleado) y nivel de ingresos (desde $1=$ menos de 1800 euros/año hasta 12 = más de 120 000 euros/año).

\section{Tasa de abandono}

Todos los participantes fueron clasificados en finaliza el tratamiento (0) si completaron el programa de intervención para maltratadores con 2 ausencias como máximo, o abandona (1) si dejaron de asistir una vez finalizada la fase de evaluación.

Problemas con el consumo de alcohol. Alcohol Use Disorders Identification Test ([AUDIT]; Babor \& Grant, 1989. Versión española de Contel, Gual, \& Colom, 1999).

El cuestionario está formado por 10 ítems en relación con el consumo de alcohol autoinformado en adultos. Las respuestas abarcan un rango de 0 (nunca) a 4 (diariamente o casi diariamente). Fue diseñado para detectar el abuso de consumo de alcohol, así como una posible dependencia. El punto de corte es igual o mayor que 8 e indica que el consumo de alcohol es peligroso y existe un riesgo de convertirse en dependencia (consistencia interna pretratamiento $=0.79 ;$ consistencia interna postratamiento $=0.78$ ).

\section{Reincidencia}

Estos datos fueron recogidos del sistema de seguimiento de los casos de VMRP del Ministerio del Interior, que incluye información de posibles reincidencias en VMRP de los agresores (López-Ossorio, González-Álvarez, \&
Andrés-Pueyo, 2016). Se consideró que existía reincidencia cuando este sistema mostraba que algún participante había realizado alguna conducta violenta contra la pareja durante el año siguiente a la finalización del tratamiento $(0=$ no existe reincidencia, $1=$ existe reincidencia).

\section{Riesgo de reincidencia}

El personal del programa, formado específicamente para ello, utilizó el protocolo Spousal Assault Risk Assessment ([SARA]; Kropp, Hart, Webster, \& Eaves, 1995; Versión española Andrés-Pueyo \& López, 2005) para evaluar el riesgo de reincidencia. Este protocolo consta de 20 ítems que reflejan los principales factores de riesgo de la VMRP. Los profesionales responden en una escala de 3 puntos $(0=$ no presente , 1 = posiblemente presente $\mathrm{y} 2=$ presente ).

\section{Atribución de responsabilidad. Intimate Partner Violence Responsibility Attribution Scale ([IPVRAS]; Lila et al., 2014).}

Es una escala autoinformada de 12 ítems con una escala de respuesta tipo Likert de 5 puntos en un rango que oscila desde 1 (completamente en desacuerdo) hasta 5 (completamente de acuerdo), cuyo objetivo es evaluar dónde sitúa el agresor la causa de su condena por VMRP. Abarca tres dimensiones: atribución de la responsabilidad al sistema legal (consistencia interna pretratamiento $=0.65$; postratamiento $=0.64)$, atribución de la responsabilidad a la víctima (consistencia interna pretratamiento $=0.66 ;$ postratamiento $=0.73$ ) y atribución de la responsabilidad al contexto personal del agresor (consistencia interna pretratamiento $=$ 0.6 ; postratamiento $=0.72$ ) .

\section{Actitudes hacia la VMRP}

La escala de gravedad percibida de violencia contra la mujer en las relaciones de pareja (Gracia et al., 2011, 2014; Lila et al., 2013) 
es una escala de 8 ítems con un formato de 10 puntos de respuesta y representa ocho situaciones hipotéticas de VMRP. El participante debe indicar qué nivel de gravedad detecta en cada una de las situaciones. Mayor puntuación indica mayor gravedad percibida de la VMRP (consistencia interna pretratamiento $=0.92$; postratamiento $=0.87$ ).

\section{Actitudes sexistas. Ambivalent Sexism Inventory (versión española de Expósito, Moya, \& Glick, 1998).}

Se trata de un inventario de 22 ítems con una escala tipo Likert de 6 puntos que oscila entre 0 ( completamente en desacuerdo ) a 5 ( completamente de acuerdo ) para medir actitudes sexistas hostiles y benevolentes. El sexismo hostil refleja actitudes explícitamente negativas hacia las mujeres (consistencia interna pretratamiento $=0.9 ;$ postratamiento $=$ 0.91), mientras que el sexismo benevolente informa de actitudes hacia las mujeres más tradicionales y paternalistas (consistencia interna pretratamiento $=0.77$; postratamiento $=0.7$ ) .

\section{Ajuste psicológico. Centre for Epidemiologic Studies Depression Scale-7 (Radloff, 1977; [CESD] versión breve, Herrero \& Gracia, 2007)}

Esta escala contiene los síntomas más comunes en la depresión. Se trata de una escala con respuestas tipo Likert de 4 puntos que oscilan entre 1 ( rara vez o nunca; menos de un día) a 4 ( todo el tiempo o la mayoría del tiempo; 5-7 días ) (consistencia interna pretratamiento $=0.8$; postratamiento $=0.77$ ).

Intimate Social Support Questionnaire (Lin, Dean, \& Ensel, 1986; versión española de Herrero, Gracia, Fuente, \& Lila, 2012). Se trata de una escala unidimensional de tres ítems que evalúa la percepción de los participantes de contar con apoyo social por parte de personas cercanas y amigos. Las respuestas se reflejan en una escala tipo Likert de 5 puntos $(1=$ La mayor parte del tiempo , 5 = nunca), donde una mayor puntuación indica mayor apoyo percibido por parte de personas cercanas (consistencia interna pretratamiento $=0.62 ;$ postratamiento $=0.6$ ) .

\section{Análisis}

En primer lugar, los participantes fueron clasificados en con problemas de consumo de alcohol (agresores con PCA) o agresores sin problemas de consumo de alcohol (agresores sin PCA), de acuerdo al criterio del test AUDIT (puntuación de corte igual o mayor a ocho). Para detectar posibles diferencias de base, se llevó a cabo una comparación entre los agresores con PCA que abandonaron la intervención y los agresores con PCA que completaron la intervención ( $n=42)$. Las diferencias entre ambos grupos se realizaron utilizando análisis de varianza univariante (ANOVA) para variables continuas y pruebas $\chi^{2}$ para variables categóricas. En segundo lugar, la relación entre abuso de alcohol y tasa de abandono ( $n=$ 107) fue analizada con una prueba $\chi^{2}$ y una regresión logística binaria con la variable abuso de alcohol como variable independiente y la tasa de abandono como variable dependiente. En tercer lugar, se llevó a cabo una prueba $\chi^{2}$ para comparar los agresores con PCA y los agresores sin PCA en tasa de reincidencia. Por último, se llevaron a cabo ANOVA de medidas repetidas para comparar los resultados de la intervención (incluido el consumo de alcohol) entre los participantes que la finalizaron, comparando las puntuaciones pre y postratamiento $(n=74)$, con la variable abuso de alcohol como variable intersujeto.

\section{Resultados}

\section{Agresores con PCA y abandono/no abandono} de la intervención

42 participantes fueron clasificados como agresores con PCA (puntuación igual o mayor a ocho) y 65 como agresores sin PCA. 
En relación con la tasa de abandono, 33 participantes causaron baja en el programa de intervención $(30.84 \%)$. No se observaron diferencias entre los agresores con PCA que finalizaron y abandonaron la intervención en variables sociodemográficas y puntuaciones pretratamiento.

\section{Tasa de abandono y abuso de alcohol}

La prueba $\chi^{2}$ para observar la relación entre abuso de alcohol y abandono de la intervención no mostró diferencias estadísticamente significativas $\left(\chi^{2}=20 ; p=\right.$ $0.65)$. La regresión logística binaria con el abuso de alcohol como variable independiente y la tasa de abandono como variable dependiente tampoco mostró una relación estadísticamente significativa $\left(\Delta \chi^{2}=0.2 ; \Delta \mathrm{df}=1 ; p=0.65 ; \mathrm{W}\right.$ $=0.2 ;$ OR $0.83 ; 95 \%$ IC 0.36-1.9). El modelo $R^{2}$ fue $<0.01$.

\section{Resultados de la intervención}

En relación con el análisis de las diferencias en los resultados de la intervención entre los participantes que la finalizaron, en primer lugar hay que señalar que no se encontraron diferencias estadísticamente significativas en reincidencia entre los participantes que completaron la intervención con PCA y sin PCA $\left(\chi^{2}=1.37\right.$; $p=0.24)$, siendo nueve $(12.16 \%)$ el número total de reincidentes (los agresores que ejercieron algún tipo de VMRP durante el año siguiente a la finalización de la intervención).

Los datos descriptivos de las variables utilizadas como resultados de tratamiento de los participantes que completaron la intervención $(n=74)$ se muestran en la Tabla 1 . Los ANOVA de medidas repetidas muestran diferencias estadísticamente significativas entre las puntuaciones pre $\mathrm{y}$ postratamiento en numerosas variables.

\section{TABLA 1}

Estadísticos descriptivos y ANOVAs de medidas repetidas $2 \times 2$ para los participantes que completan la intervención $(n=74)$

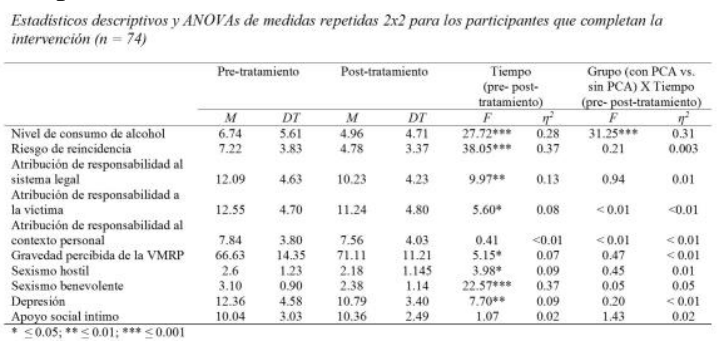

Fuente: elaboración propia

En primer lugar, se observa una reducción significativa del consumo de alcohol en los agresores con PCA que han completado el programa de intervención para maltratadores, a pesar de que el programa no aborda específicamente el PCA. El ANOVA de medidas repetidas muestra un efecto significativo en la interacción Grupo (agresores con PCA vs. Agresores sin PCA) $x$ Tiempo (pretratamiento vs. postratamiento) en relación con el consumo de alcohol. El grupo de agresores con PCA mostró puntuaciones en consumo del alcohol significativamente inferiores tras el tratamiento $(\mathrm{M}[\mathrm{DE}]$ pretratamiento $=12.93[3.8] ; M[\mathrm{DE}]$ postratamiento $=7.85[4.74](F=46.75 ; p$ $\left.<0.001 ; \chi^{2}=0.4\right)$, pero no se observan diferencias significativas entre las puntuaciones pretratamiento y postratamiento en consumo de alcohol en el grupo de agresores sin PCA $(\mathrm{M}[\mathrm{DE}]$ pretratamiento $=3.11[2.36] ; \mathrm{M}[\mathrm{DE}]$ postratamiento $=3.26[3.81](F=0.07 ; p=0.79)$ (Figura 1). 


\section{Figura 1}

Efecto de interacción Tiempo (pre- vs. posttratamiento) $x$ Grupo (con PCA vs. sin PCA) en consumo de alcohol

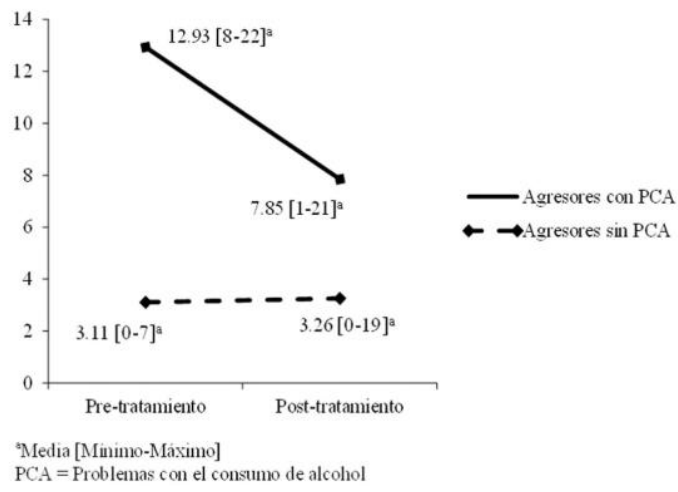

Fuente: elaboración propia

Por otra parte, tras la intervención, los participantes que finalizaron el tratamiento mostraron menor riesgo de reincidencia, menor atribución de la responsabilidad al sistema legal y a la víctima, mayor gravedad percibida de la VMRP, menor sexismo hostil, menor sexismo benevolente y menores síntomas depresivos. El efecto de las interacciones no fue significativo (Tabla 1), mostrando que no existen diferencias en los resultados de la intervención entre agresores con PCA y agresores sin PCA. Los resultados revelan que, a pesar de los PCA, los participantes que completan la intervención muestran cambios positivos en las variables analizadas.

\section{Discusión}

Las mayores tasas de VMRP y de PCA señalan a los inmigrantes latinoamericanos derivados a programas de intervención con maltratadores en España como un colectivo que requiere de una especial atención. En este sentido, los resultados de este estudio proporcionan información relevante para la intervención. El primer objetivo de este trabajo fue comprobar, en un grupo de inmigrantes latinoamericanos condenados por VMRP, si los participantes que tienen PCA presentan mayor probabilidad de abandono en un programa de intervención para maltratadores que no trata específicamente el PCA. Numerosos estudios previos ponen de manifiesto que el PCA es uno de los predictores del abandono de los programas de intervención con maltratadores (Catalá-Miñana, Lila, Conchell, Romero-Martínez, \& MoyaAlbiol, 2013; Hilton \& Harris, 2005; Olver et al., 2011). Sin embargo, los resultados de esta investigación indican que, en el caso de los inmigrantes latinoamericanos en España, estas diferencias no son significativas, de manera que no puede afirmarse que se produzca mayor abandono de la intervención por parte de aquellos que tienen PCA.

Estos resultados hay que tomarlos con cautela, en la medida en que el tamaño de la muestra es relativamente pequeño. Más allá del tamaño de la muestra, otra posible explicación para este resultado podría hallarse en la elevada normalización del consumo abusivo de alcohol entre los inmigrantes latinoamericanos (Conde \& Herranz, 2004; Tortajada et al., 2008). Al no ser un consumo que ellos mismos y su entorno interpreten como problemático, pudiera ser que, solo en los casos extremos, el consumo de alcohol tuviera un efecto sobre el abandono. Quizá con este colectivo el criterio utilizado normalmente en el AUDIT para señalar la existencia de problemas de consumo abusivo de alcohol (alcanzar una puntuación igual o mayor que ocho) no sea suficiente para detectar a aquellos individuos que, entre el conjunto de inmigrantes latinoamericanos, tengan problemas de consumo de alcohol que realmente les impida su desempeño cotidiano, diferenciándolos de aquellos que beben de una manera socialmente aceptada y no impeditiva. Sería conveniente realizar futuros estudios que permitan conocer con más detalle cuáles son las causas de la ausencia de relación entre PCA y abandono de la intervención en el caso de los participantes latinoamericanos en los programas de intervención con maltratadores.

El segundo objetivo fue analizar si los resultados de la intervención entre los inmigrantes latinoamericanos con PCA difieren de aquellos que no tienen PCA. El porcentaje de reincidentes en este estudio es similar al 
El consumo abusivo de alcohol en inmigrantes latinoamericanos participantes en programas de i...

obtenido en trabajos previos en los que se analiza la reincidencia durante el año posterior a la finalización de la intervención (Eckhardt, Holtzworth-Munroe, Norlander, Sibley, \& Cahill, 2008; Kingsnorth, 2006; Lin et al., 2009; Loinaz, 2014), aunque no se encontraron diferencias significativas entre los participantes con PCA y los que no tienen PCA.

En relación con el resto de variables incluidas en el estudio hay que subrayar que, para el total de la muestra, se produjeron cambios significativos entre el principio y el final de la intervención en casi todas ellas. Se observa una reducción significativa en el riesgo de reincidencia, la atribución de responsabilidad (al sistema legal y a la víctima), el sexismo hostil y benevolente y la sintomatología depresiva. Además, se observa un aumento de la gravedad percibida de la VMRP. Todas estas variables han sido relacionadas con la VMRP, de forma que los cambios observados pueden contribuir a la reducción de la misma (e. g., Colvin, Cullen, \& Vander Ven, 2002; Coulter \& Vande Weerd, 2009; Dankwort \& Austin, 1999; Lila et al., 2013; Novo et al., 2012; Scott \& Straus, 2007; Wallach \& Sela, 2008). Hay que señalar que las interacciones entre el tiempo de intervención (pre vs. postratamiento) $\mathrm{y}$ el grupo de pertenencia (PCA vs. No PCA) no son significativas para ninguna de las variables analizadas, con excepción de la variable consumo de alcohol. Así, los participantes que finalizan la intervención se benefician del programa en términos de las variables evaluadas, independientemente de si pertenecen o no al grupo de inmigrantes con PCA.

En cuanto a qué sucede en el caso del consumo de alcohol entre los participantes con PCA que finalizan el programa, los resultados del presente estudio muestran una reducción significativa de los problemas derivados del consumo abusivo, incluso aunque no se haya realizado una intervención destinada a tratar este problema de manera específica. Este resultado se encuentra en la línea de los planteamientos de Bennett (2008), quien señala que los programas de intervención con maltratadores y los tratamientos para el consumo abusivo de alcohol comparten actividades y se focalizan en variables similares (e. g., ira, estrés, etc.). Igualmente, ambos tipos de intervención normalmente son grupales y se centran en el cambio de actitudes y la prevención de la recaída/reincidencia. Dadas estas características compartidas, podríamos esperar que las intervenciones con maltratadores redujeran los PCA en aquellos participantes que finalizan la intervención, suposición que se ve confirmada en esta investigación.

Este resultado puede deberse a diversas causas tales como, por ejemplo, la guía y supervisión de los terapeutas, el aprendizaje de alternativas saludables para la resolución de los conflictos y/o el apoyo del grupo y del terapeuta en el programa de intervención con maltratadores. Aunque la reducción del consumo de alcohol no es en sí mismo un objetivo de la intervención, se podría estar influyendo en esta conducta indirectamente (por ejemplo, a partir de la norma existente en el programa de no acudir a las sesiones de intervención habiendo consumido alcohol u otras substancias, o si los terapeutas aconsejan al participante de manera informal en relación a su problema de consumo). Aunque los datos no permitan identificar los factores que subyacen a la reducción en el consumo de alcohol, el hecho de que esto ocurra entre los maltratadores inmigrantes latinoamericanos con PCA que finalizan el programa de intervención, es un resultado que se debe tener en cuenta en el debate existente acerca de si se incrementa la efectividad de los programas cuando se incluye en los mismos un tratamiento específico para el PCA (e. g., Crane et al., 2015; Stuart et al., 2013; Wilson, Graham, \& Taft, 2014). El hecho de que, sin implementar un tratamiento específico, el consumo de alcohol se reduzca entre los participantes del presente estudio, subraya el efecto que tienen los programas de intervención con maltratadores sobre la conducta de los participantes, impacto que debería controlarse en los trabajos que analizan la efectividad de las intervenciones que combinan tratamiento de alcohol y de violencia.

Este estudio tiene algunas limitaciones. En primer lugar, tal y como ya se ha señalado, 
sería recomendable replicarlo con una muestra más amplia. En segundo lugar, algunas de las medidas utilizadas fueron autoinformes, de manera que los resultados están sujetos a las limitaciones que tienen estos instrumentos en este tipo de población. Lo mismo puede decirse en relación con los instrumentos que medían el consumo de alcohol. Si se incluyeran medidas psicofisiológicas o medidas que los participantes no puedan manipular (e. g., medidas implícitas de las actitudes; Gracia, Rodriguez, \& Lila, 2015), los resultados podrían variar.

En tercer lugar, la reincidencia se obtuvo a partir de fuentes oficiales. El porcentaje de reincidencia informado por la víctima podría ser mayor (Williams \& Houghton, 2004); sin embargo, en España, los servicios que derivan los penados a los programas de intervención comunitarios no proporcionan la información sobre las víctimas, por lo que no es posible el acceso a las mismas con fines de investigación (Bolivar \& Vanfraechem, 2015; Sierra, Bermúdez, Buela-Casal, Salinas, \& Monge, 2014). Por último, no se dispone de los datos de los participantes que han abandonado el programa en el momento temporal correspondiente a la evaluación postratamiento, lo que podría incrementar la fiabilidad de los resultados.

A pesar de estas limitaciones, el trabajo se centra en un colectivo, el de los inmigrantes latinoamericanos, poco estudiado en este ámbito y que presentan unas características que podrían llevarles a ser especialmente resistentes al cambio (elevados niveles de VMRP y de PCA). El que no se hayan encontrado diferencias entre los grupos de inmigrantes latinoamericanos con PCA y sin PCA en las variables resultado analizadas, junto a la reducción de los PCA, apoya el punto de vista mantenido por algunos autores de que para buena parte de los participantes en los programas de intervención con maltratadores no hay necesidad de realizar una adaptación específica para que los participantes se beneficien de ellos (e. g., Gondolf, 2012). Este resultado es particularmente relevante en países como España, donde los recursos disponibles para la intervención con maltratadores son aún muy escasos. Tal y como señalan los resultados, para incrementar la eficacia de los programas se debería prestar una especial atención a la mejora de las estrategias que incrementen la adherencia a la intervención entre los participantes inmigrantes latinoamericanos (con o $\sin$ PCA).

\section{Referencias}

Abramsky, T., Watts, C. H., Garcia-Moreno, C., Devries, K., Kiss, L., Ellsberg, M., ... Heise, L. (2011). What factors are associated with recent intimate partner violence? Findings from the WHO multicountry study on women's health and domestic violence. BMC Public Health, 11 , 109. http://dx.doi.org/10.1186/1471-2458 -11-109

Andrés-Pueyo, A., \& Echeburúa, E. (2010). Valoración del riesgo de violencia: instrumentos disponibles e indicaciones de aplicación. Psicothema , 22(3), 403-409.

Andrés-Pueyo, A., \& López, S. (2005). Manual para la valoración del riesgo de reincidencia de violencia contra la pareja . Barcelona: Publicacions y Edicions de la Universitat de Barcelona.

Arias, E., Arce, R., \& Vilariño, M. (2013). Batterer intervention programmes: A meta-analytic review of effectiveness. Psychosocial Intervention, $22,153-160 . \mathrm{h}$ ttp://dx.doi.org/10.5093/in2013a18

Arteaga, A., López-Goñi, J. J., \& FernándezMontalvo, J. (2015). Differential profiles of drug-addicted patients according to gender and the perpetration of intimate partner violence. Drug and Alcohol Dependence, 155 , 183-189. http://dx.doi.org/10.1016/j. drugalcdep.2015.07.018

Babcock, J. C., Green, C. E., \& Robie, C. (2004). Does batterers' treatment work? A meta-analytic review of domestic violence treatment. Clinical Psychology Review, 23 , 1023-1053. http://dx.doi.org/10.1016/j.cp r.2002.07.001 
Babor, T. E., \& Grant, M. G. (1989). From clinical research to secondary prevention: International collaboration in the development of the Alcohol Use Disorders Identification Test (AUDIT). Alcohol Health \& Research World, 13 , 371-374.

Bennett, L. W. (2008). Substance abuse by men in partner abuse intervention programs: Current issues and promising trends. Violence and Victims, 23 , 236-248. http:// dx.doi.org/10.1891/0886-6708.23.2.236

Bennett, L. W., Stoops, C., Call, C., \& Flett, H. (2007). Program completion and re-arrest in a batterer intervention system. Research on Social Work Practice, 17 , 42-54. http:/ /dx.doi.org/10.1177/1049731506293729

Bowen, E. (2011). The rehabilitation of partnerviolent men . Oxford: Wiley-Blackwell.

Bolivar, D., \& Vanfraechem, I. (2015). Víctimas en justicia restaurativa: ¿sujetos activos $o$ en necesidad? Un estudio europeo desde la perspectiva de operadores sociales. Universitas Psychologica, 14 , 1437-1458. http://dx.doi.org/10.11144/Jav eriana.up14-4.vjrs

Camacho, J. M., \& Comas, D. (2003). El ocio y los jóvenes inmigrantes. Estudios de Juventud, 60 , 63-88.

Carbajosa, P., \& Boira, S. (2013). Estado actual y retos futuros de los programas para hombres condenados por violencia de género en España. Psychosocial Intervention, 22 , 145-152. http://dx.doi.or g/10.5093/in2013a17

Carbajosa, P., Lila, M., Negredo, L., \& Pérez, M. (2011). El delito de violencia de género y los penados extranjeros . Madrid: Ministerio del Interior. Recuperado de http://www.interior.gob.es/file/53/5372 3/53723.pdf

Catalá-Miñana, A., Lila, M., Conchell, R., Romero-Martínez, A., \& Moya-Albiol, L. (2013). ¿Se benefician de los programas de intervención que no tratan específicamente el consumo de alcohol los maltratadores con problemas de consumo abusivo?
Psychosocial Intervention, 22 , 135-143. h ttp://dx.doi.org/10.5093/in2013a16

Catalá-Miñana, A., Lila, M., \& Oliver, A. (2013). Consumo de alcohol en hombres penados por violencia contra la pareja: factores individuales y contextuales. Adicciones, 25 (1), 19-28.

Cho, H., Velez-Ortiz, D., \& Parra-Cardona, J. (2014). Prevalence of intimate partner violence and associated risk factors among Latinos/as: An exploratory study with three Latino subpopulations. Violence Against Women, 20 , 1041-1058. http://dx.doi.org/ $10.1177 / 1077801214549636$

Colvin, M., Cullen, F. T., \& Vander Ven, T. (2002). Coercion, social support, and crime: An emerging theoretical consensus. Criminology, 40 , 19-42. http://dx.doi.org/ 10.1111/j.1745-9125.2001.tb00948.x

Conde, F., \& Herranz, D. (2004). Los procesos de integración de los inmigrantes. Pautas de consumo de alcohol y modelos culturales de referencia. Madrid: Fundación CREFAT.

Contel, M., Gual, A., \& Colom, J. (1999). Test para la identificación de trastornos por uso de alcohol (AUDIT): traducción y validación del AUDIT al catalán y castellano. Adicciones, 11 (4), 337-347.

Contreras, L. (2014). Factores de riesgo de homicidio a la mujer en la relación de pareja. Universitas Psychologica, 13, 681-692. http://dx.doi.org/10.11144/Javeri ana.UPSY13-2.frhm

Coulter, M., \& VanderWeerd, C. (2009). Reducing domestic violence and other criminal recidivism: Effectiveness of a multilevel batterers intervention program. Violence and Victims, 24 , 139-152. http:// dx.doi.org/10.1891/0886-6708.24.2.139

Crane, C. A., Eckhardt, C. I., \& Schlauch, R. C. (2015). Motivational enhancement mitigates the effects of problematic alcohol use on treatment compliance among partner violent offenders: Results of a randomized clinical trial. Journal of Consulting and Clinical Psychology, 83 , 689-695. http://d x.doi.org/10.1037/a0039345 
Marisol Lila, Enrique Gracia, Alba Catalá-Miñana, Faraj A. Santirso, Et al.

Daly, J. E., \& Pelowski, S. (2000). Predictors of dropout among men who batter: A review of studies with implications for research and practice. Violence and Victims, 15 (2), 137-160.

Dankwort, J., \& Austin, J. (1999). Standards for batterer treatment programs in Canada: A history and review. Canadian Journal of Community Mental Health, 18 , 19-38. http ://dx.doi.org/10.7870/cjcmh-1999-0002

Devries, K. M., Mak, J. Y., García-Moreno, C., Petzold, M., Child, J. C., Falder, G., ... Watts, C. H. (2013). The global prevalence of intimate partner violence against women. Science, 340, 1527-1528. http://dx.doi.org/10.1126/science.1240937

Echauri, J. A., Fernández-Montalvo, J., Martínez, M., \& Azkarate, J. M. (2013). Effectiveness of a treatment programme for inmigrants who committed gender-based violence against their partner. Psicothema, 25 , 49-54. http://dx.doi.org/10.7334/psico thema2012.75

Eckhardt, C., Holtzworth-Munroe, A., Norlander, B., Sibley, A., \& Cahill, M. (2008). Readiness to change, partner violence subtypes, and treatment outcomes among men in treatment for partner assault. Violence and Victims, 23 , 446-475. http:// dx.doi.org/10.1891/0886-6708.23.4.446

Eckhardt, C. I., Murphy, C. M., Whitaker, D. J., Sprunger, J., Dykstra, R., \& Woodard, K. (2013). The effectiveness of intervention programs for perpetrators and victims of intimate partner violence. Partner Abuse, 4 , 196-231. http://dx.doi.org/10.1891/19466560.4.2.e17

European Union Agency for Fundamental Rights. (2014). Violence against women: An EU-wide survey . Luxembourg: Publications Office of the European Union. Recuperado de http://fra.europa.eu/en/publ ication/2014/vaw-survey-main-results

Expósito, F., Moya, M., \& Glick, P. (1998). Sexismo ambivalente: medición y correlatos. Revista de Psicología Social, 13 , 159-169. http://dx.doi.org/10.1174/02134 7498760350641
Feder, L., \& Wilson, D. B. (2005). A meta-analytic review of court-mandated batterer intervention programs: Can courts affect abusers' behavior? Journal of Experimental Criminology, 1 , 239-262. http://dx.doi.org/10.1007/s11292 $-005-1179-0$

Foran, H. M., \& O'Leary, K. D. (2008). Alcohol and intimate partner violence: A meta-analytic review. Clinical Psychology Review, 28 , 1222-1234. http://dx.doi.org/1 0.1016/j.cpr.2008.05.001

Gil-González, D., Vives-Cases, C., ÁlvarezDardet, C., \& Latour-Pérez, J. (2006). Alcohol and intimate partner violence: Do we have enough information to act? European Journal of Public Health, 16 , 278-284. http://dx.doi.org/10.1093/eurpub/ ck1016

Glick, P., Sakalli-Ugurlu, N., Ferreira, M. C., \& de Souza, M. A. (2002). Ambivalent sexism and attitudes toward wife abuse in Turkey and Brazil. Psychology of Women Quarterly, 26 , 292-297. http://dx.doi.org/1 0.1111/1471-6402.t01-1-00068

Gondolf, E. W. (2012). The future of batterer programs: Reassessing evidence-based practice. Boston: Northeastern University Press.

Gracia, E., García, F., \& Lila, M. (2011). Police attitudes toward policing partner violence against women: Do they correspond to different psychosocial profiles? Journal of Interpersonal Violence, $26,189-207 . \mathrm{http}$ : //dx.doi.org/10.1177/0886260510362892

Gracia, E., García, F., \& Lila, M. (2014). Male police officers' law enforcement preferences in cases of intimate partner violence versus non-intimate interpersonal violence do sexist attitudes and empathy matter? Criminal, Justice and Behavior, 41 , 1195-1213. http://dx.doi.org/10.1177/009 3854814541655

Gracia, E., López-Quílez, A., Marco, M., Lladosa, S., \& Lila, M. (2014). Exploring neighborhood influences on small-area variations in intimate partner violence risk: A Bayesian random-effects 
modeling approach. International Journal of Environmental Research and Public Health, 11 , 866-882. http://dx.doi.org/10.3 390/ijerph110100866

Gracia, E., López-Quílez, A., Marco, M., Lladosa, S., \& Lila, M. (2015). The spatial epidemiology of intimate partner violence: Do neighborhoods matter? American Journal of Epidemiology, 182, 58-66. http ://dx.doi.org/10.1093/aje/kwv016

Gracia, E., Rodriguez, C. M., \& Lila, M. (2015). Preliminary evaluation of an analog procedure to assess acceptability of intimate partner violence against women: The Partner Violence Acceptability Movie Task . Frontiers in Psychology, 6, 1567. h ttp://dx.doi.org/10.3389/fpsyg.2015.01567

Gracia, E., \& Tomás, J. M. (2014). Correlates of victim-blaming attitudes regarding partner violence against women among the Spanish general population. Violence Against Women, 20 , 26-41. http://dx.doi.o rg/10.1177/1077801213520577

Graham, K., Bernards, S., Knibbe, R., Kairouz, S., Kuntsche, S., Wilsnack, S. C., ... Gmel, G. (2011). Alcohol\#related negative consequences among drinkers around the world. Addiction, 106 , 1391-1405. http://dx.doi.org/10.1111/j.136 0-0443.2011.03425.x

Heise, L. L. (2011). What works to prevent partner violence?: An evidence overview . London: STRIVE Research Consortium, London School of Hygiene and Tropical Medicine.

Henning, K., \& Holdford, R. (2006). Minimization, denial, and victim blaming by batterers: How much does the truth matter? Criminal Justice and Behavior, 33 , 110-130. http://dx.doi.org/10.1177/00938 54805282322

Herrero, J., \& Gracia, E. (2007). Una medida breve de la sintomatología depresiva (CESD 7). Salud Mental, 30 , 40-46.

Herrero, J., Gracia, E., Fuente, A., \& Lila, M. (2012). Desorden social, integracio\#n social y bienestar subjetivo en inmigrantes latinoamericanos en Espan\#a. Anales de
Psicologi\#a, 28 , 505-514. http://dx.doi.or g/10.6018/analesps.28.2.148721

Hilton, N. Z., \& Harris, G. T. (2005). Predicting wife assault: A critical review and implications for policy and practice. Trauma, Violence, \& Abuse, 6, 3-23. http: //dx.doi.org/10.1177/154838004272463

Jewell, L. M., \& Wormith, J. S. (2010). Variables associated with attrition from domestic violence treatment programs targeting male batterers: A meta-analysis. Criminal Justice and Behavior, 37 , 1086-1113. http://dx.doi.org/10.1177/0093 854810376815

Jewkes, R. (2002). Intimate partner violence: Causes and prevention. Lancet, 359 , 1423-1429. http://dx.doi.org/10.1016/S014 0-6736(02)08357-5

Kingsnorth, R. (2006). Intimate partner violence predictors of recidivism in a sample of arrestees. Violence Against Women, 12 , 917-935. http://dx.doi.org/10.1177/107780 1206293081

Kropp, P. D., Hart, S., Webster, C., \& Eaves, D. (1995). The spousal assault risk assessment guide . Vancouver: BCAFV.

Langenderfer, L. (2013). Alcohol use among partner violent adults: Reviewing recent literature to inform intervention. Aggression and Violent Behavior, 18 , 152-158. http://dx.doi.org/10.1016/j.avb.2 012.11 .013

Lila, M. (2013). La intervención con hombres condenados por violencia de pareja contra la mujer en España: investigación y avances en intervención. Psychosocial Intervention, 22 , 81-85. http://dx.doi.org/1 0.5093/in2013a10

Lila, M., Gracia, E., \& García, F. (2013). Ambivalent sexism, empathy, and law enforcement attitudes towards partner violence against women among male police officers. Psychology, Crime \& Law, 19 , 907-919. http://dx.doi.org/10.1080/106831 6X.2012.719619

Lila, M., Gracia, E., \& Herrero, J. (2012). Asunción de responsabilidad en hombres maltratadores: influencia de la autoestima, 
la personalidad narcisista y la personalidad antisocial. Revista Latinoamericana de Psicología, 44, 99-108.

Lila, M., Gracia, E., \& Murgui, S. (2013). Psychological adjustment and victimblaming among intimate partner violence offenders: The role of social support and stressful life events. European Journal of Psychology Applied to Legal Context, 5 , 147-153. http://dx.doi.org/10.5093/ejpalc2 $013 \mathrm{a} 4$

Lila, M., Oliver, A., Catalá-Miñana, A., \& Conchell, R. (2014). Recidivism risk reduction assessment in batterer intervention programs: A key indicator for program efficacy evaluation. Psychosocial Intervention, 23 , 217-223. http://dx.doi.or $\mathrm{g} / 10.1016 / \mathrm{j} . p s i .2014 .11 .001$

Lila, M., Oliver, A., Catalá-Miñana, A., Galiana, L., \& Gracia, E. (2014). The intimate partner violence responsibility attribution scales (IPVRAS). European Journal of Psychology Applied to Legal Context, 6 , 29-36. http://dx.doi.org/10.5093/ejpalc201 $4 \mathrm{a} 4$

Lila, M., Oliver, A., Galiana, L., \& Gracia, E. (2013). Predicting success indicators of an intervention programme for convicted intimate-partner violence offenders: The Contexto Programme. European Journal of Psychology Applied to Legal Context, 5 (1), 73-95.

Lila, M., Oliver, A., Lorenzo, M. V., \& Catalá, A. (2013). Valoración del riesgo de reincidencia en violencia contra la mujer en las relaciones de pareja: importancia del apoyo social. Revista de Psicología Social, 28 , 225-236. doi: http://dx.doi.org/10.117 4/021347413806196681

Lin, N., Dean, A., \& Ensel, W. (1986). Social support, life events, and depression. New York: Academic Press.

Lin, S. C., Su, C. Y., Chou, F. H. C., Chen, S. P., Huang, J. J., Wu, G. T. E., \& Chen, C. C. (2009). Domestic violence recidivism in high-risk Taiwanese offenders after the completion of violence treatment programs. Journal of Forensic
Psychiatry \& Psychology, 20 , 458-472. doi: http://dx.doi.org/10.1080/1478994080 2638341

Loinaz, I. (2014). Typologies, risk and recidivism in partner-violent men with the B-SAFER: A pilot study. Psychology, Crime \& Law, 20 , 183-198. doi: http://dx. doi.org/10.1080/1068316X.2013.770854

López-Ossorio, J. J., González-Álvarez, J. L., \& Andrés-Pueyo, A. (2016). Eficacia predictiva de la valoración policial del riesgo de la violencia de género. Psychosocial Intervention, 25 , 1-7. doi: ht tp://dx.doi.org/10.1016/j.psi.2015.10.002

Ministerio de Sanidad, Servicios Sociales e Igualdad. (2011). Macroencuesta de violencia de género 2011 . Recuperado de http://www.observatorioviolencia.org/u pload_images/File/DOC1329745747_mac roencuesta2011_principales_resultados-1. pdf

Novo, M., Fariña, F., Seijo, M. D., \& Arce, R. (2012). Assessment of a community rehabilitation programme in convicted male intimate-partner violence offenders. International Journal of Clinical and Health Psychology, 12 (2), 219-234.

Observatorio contra la Violencia Doméstica y de Género, Consejo General del Poder Judicial. (2014). Datos de denuncias, procedimientos penales $y$ civiles registrados, órdenes de protección solicitadas en los juzgados de violencia sobre la mujer y sentencias dictadas por los órganos jurisdiccionales en esta materia. Recuperado de http://www.poderjudicial.es/cgpj/es/Te mas/Violencia_domestica_y_de_genero/A ctividad_del_Observatorio/Datos_estadisti $\cos /$

Olver, M. E., Stockdale, K. C., \& Wormith, J. S. (2011). A meta-analysis of predictors of offender treatment attrition and its relationship to recidivism. Journal of Consulting and Clinical Psychology, 79, 6-21. http://dx.doi.org/10.1037/a0022200

Organización Mundial de la Salud. (2010). Preventing intimate partner and sexual 
violence against women: Taking action and generating evidence . Ginebra: Autor.

Organización Mundial de la Salud. (2013). Global and regional estimates of violence against women: Prevalence and health effects of intimate partner violence and non-partner sexual violence. Ginebra: Autor.

Parra-Cardona, J. R., Escobar-Chew, A. R., Holtrop, K., Carpenter, G., Guzmán, R., Hernández, D., ... González, D. (2013). "En el grupo tomas conciencia (In group you become aware)": Latino inmigrants ' satisfaction with a culturally informed intervention for men who better. Violence Against Women, 19 , 107- 132. http://dx.do i.org/10.1177/1077801212475338

Radloff, L. S. (1977). The CES-D Scale: A selfreport depression scale for research in the general population. Applied Psychological Measurement , 1, 385-401. http://dx.doi.or $\mathrm{g} / 10.1177 / 014662167700100306$

Rondeau, G., Brodeur, N., Brochu, S., \& Lemire, G. (2001). Dropout and completion of treatment among spouse abusers. Violence and Victims, 16 (2), 127-143.

Sabina, C., Cuevas, C. A., \& Lannen, E. (2014). The likelihood of Latino women to seek help in response to interpersonal victimization: An examination of individual, interpersonal and sociocultural influences. Psychosocial Intervention, 23 , 95-103. http://dx.doi.org /10.1016/j.psi.2014.07.005

Scott, K., King, C., McGinn, H., \& Hosseini, N. (2011). Effects of motivational enhancement on immediate outcomes of batterer intervention. Journal of Family Violence, 26 , 139-149. http://dx.doi.org/1 0.1007/s10896-010-9353-1

Scott, K., \& Straus, M. (2007). Denial, minimization, partner blaming, and intimate aggression in dating partners. Journal of Family Violence, 22 , 851-871. http://dx.doi.org/10.1177/088626 0507301227

Sierra, J. C., Bermúdez, M. P., Buela-Casal, G., Salinas, J. M., \& Monge, F. S. (2014).
Factors associated with the intimate partner violence and their complaint in a sample of women. Universitas Psychologica, 13 , 37-46. http://dx.doi.org/10.11144/Javerian a.UPSY13-1.vaea

Stuart, G. L., O'Farrell, T. J., \& Temple, J. R. (2009). Review of the association between treatment for substance misuse and reductions in intimate partner violence. Substance Use \& Misuse, 44 , 1298-1317. http://dx.doi.org/10.1080/1082 6080902961385

Stuart, G. L., Shorey, R. C., Moore, T. M., Ramsey, S. E., Kahler, C. W., O'Farrell, ... Monti, P. M. (2013). Randomized clinical trial examining the incremental efficacy of a 90-minute motivational alcohol intervention as an adjunct to standard batterer intervention for men. Addiction, 108 , 1376-1384. http://dx.doi.o rg/10.1111/add.12142

Sussman, S. (2005). Prevention of adolescent alcohol problems in special populations. Recent Developments in Alcoholism, 17 , 225-253. http://dx.doi.org/10.1007/0-306 -48626-1_11

Tortajada, S., Valderrama, J. C., Castellano, M., Llorens, N., Agulló, V., Herzog, B., \& Aleixandre, R. (2008). Consumo de alcohol y su percepción por parte de inmigrantes latinoamericanos. Psicothema, 20 (3), 403-407.

Unger, J. B. (2015). Preventing substance use and misuse among racial and ethnic minority adolescents: Why are we not addressing discrimination in prevention programs? Substance Use \& Misuse, 50 , 952-955. http://dx.doi.org/0.3109/1082608 4.2015.1010903

Vargas, V., Lila, M., \& CataláMiñana, A. (2015). ¿Influyen las diferencias culturales en los resultados de los programas de intervención con maltratadores? Un estudio con agresores españoles y latinoamericanos. Psychosocial Intervention, 24 , 41-47. http ://dx.doi.org/10.1016/j.psi.2015.03.001 
Vaughn, M., Salas-Wright, C. P., Cooper-Sadlo, S., Maynard, B. R., \& Larson, M. (2014). Are immigrants more likely than nativeborn Americans to perpetrate intimate partner violence. Journal of Interpersonal Violence, 30 , 1888-1907. http://dx.doi.org /10.1177/0886260514549053

Wallach, H. S., \& Sela, T. (2008). The importance of male batters' attributions in understanding and preventing domestic violence. Journal of Family Violence, 23 (7), 655-660.

Williams, K. R., \& Houghton, A. B. (2004). Assessing the risk of domestic violence reoffending: A validation study. Law and Human Behavior, 28 , 437-455. http://dx.doi.org/10.1023/B:LAH U.0000039334.59297.f0

Wilson, I. M., Graham, K., \& Taft, A. (2014). Alcohol interventions, alcohol policy and intimate partner violence: A systematic review. BMC Public Health, 14 , 881. http ://dx.doi.org/0.1186/1471-2458-14-881

\section{Notas}

* Artículo de investigación 